\title{
BOLES HELENÍSTICOS CON RELIEVES A MOLDE EN EL SANTUARIO DE CALESCOVES (MENORCA)
}

\section{HELENISTIC MOLDMADE RELIEF BOWLS FROM THE CALESCOVES SANCTUARY (MINORCA)}

\author{
ELENA SÁNCHEZ LÓPEZ* \\ MARGARITA ORFILA PONS**
}

Resumen: Un proyecto de investigación recientemente desarrollado en Calescoves (Alaior, Menorca), y en concreto las excavaciones llevadas a cabo en la Cova dels Jurats, han supuesto la documentación de un interesante conjunto de materiales cerámicos, en el que conviven elementos de producción local e importaciones. De estos últimos analizamos aquí un conjunto muy significativo, el perteneciente a los boles helenísticos procedentes de la zona jonia. Vinculados en su zona de origen al consumo de vino, la presencia de estos vasos en el santuario menorquín debe vincularse a los rituales y libaciones que se debieron llevar a cabo en la cueva.

Palabras clave: Arqueología romana, cerámica helenística, Islas Baleares, santuario rupestre, Mar Mediterráneo, contactos comerciales.

\section{INTRODUCCIÓN}

Objeto de estudio de un Proyecto de Investigación desarrollado desde las Universidades de Granada, Barcelona y Macerata entre 2010 y 2012 (Orfila et al. 2010 y 2013), el santuario de la "Cova dels Jurats o Esglesia", perteneciente al Conjunto Arqueológico de Calescoves

* Investigadora Postdoctoral. Grupo de Investigación HUM296. Dpto Prehistoria y Arqueología. Facultad de Filosofía y Letras. Campus de Cartuja s/n. Universidad de Granada. 18071 Granada. Correo-e: elenasanchez@ugr.es

\begin{abstract}
The research project recently carried out in Calescoves (Alaior, Menorca), and in particular the excavation in the Cova dels Jurats, has allowed us to study an interesting collection of ceramics, including both local and imported items. We will analyse here the Hellenistic bowls from the Ionian area. Linked in their original area to the consumption of wine, the presence of these vessels in this sanctuary must be linked to the rituals and libations that were due to perform in the cave.

Key words: Roman archaeology, Hellenistic pottery, Balearic Islands, rock sanctuary, Mediterranean Sea, trading contacts.
\end{abstract}

(BIC R-I-51-0003150-00000), se localiza en la cala del mismo nombre (TM de Alaior), una unidad formada por la confluencia de los barrancos de Lloc Nou d'es Fasser, Biniadris y Sant Domingo, siendo estos dos últimos los que le confieren su peculiar forma en $\mathrm{Y}$, con dos fondos y una sola boca de entrada, origen del topónimo plural por el que es conocida. (Fig. 1).

** Catedrática de Arqueología. Grupo de Investigación HUM296. Dpto Prehistoria y Arqueología. Facultad de Filosofía y Letras. Campus de Cartuja s/n. Universidad de Granada. 18071 Granada. Correo-e: orfila@ugr.es 


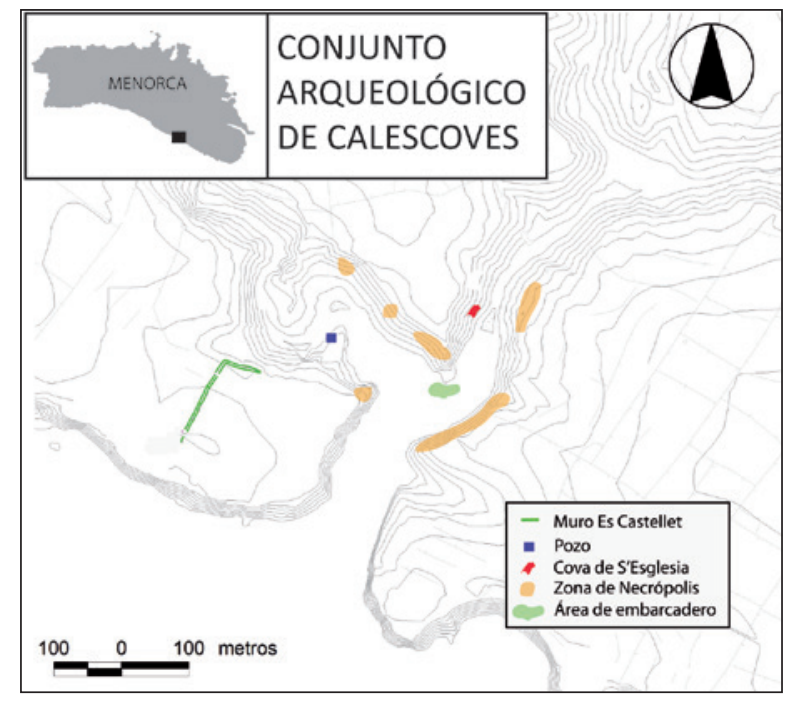

Figura 1. Localización de Calescoves y elementos del Conjunto Arqueológico.

El conjunto comenzó a ser analizado de forma sistemática a partir de 1966, cuando Cristóbal Veny inició las excavaciones arqueológicas que permitieron establecer la dimensión real de la necrópolis hipogeica que cubre las altas paredes de los acantilados de la cala, en uso entre los siglos IX y II a.C. (Veny 1982; Gornés y Gual 2000; Gornés 1997 y 2000). Entre los elementos de cronología prehistórica documentados en Calescoves se encuentran también el asentamiento costero de Es Castellet (Ramis 1817: 87; Plantalamor 1991; Sánchez et al. 2013), ubicado en el lateral oeste de la boca de entrada, y un pozo ritual con escalinata de acceso (Veny 1982: 22, Lam. LXII y LXIII; Plantalamor 1991: $560)$, tipología documentada en contextos talayóticos en otros puntos de la isla (Sánchez et al. 2013).

La localización y la morfología de Calescoves convierten además el enclave en un fondeadero perfecto, funcionalidad que aún desempeña en la actualidad pero que también ha sido demostrada por el registro arqueológico para la Antigüedad. Según los resultados del proyecto desarrollado por Fernández-Miranda, este fue su uso desde el siglo IV a.C., con una actividad especialmente importante durante los siglos III y II a.C., y una frecuentación que se convertiría en esporádica a partir del siglo I a.C. y hasta el VII d.C. (Fernández-Miranda et al. 1977; Belén y Fernández-Miranda 1979; Fernández-Miranda y Rodero 1991).

Tal vez vinculado a este uso como fondeadero se encontrara el denominado santuario del Coberxo Blanc. Compuesto de un total de siete recortes destinados a la fijación de estatuas, signa o arulae, que desde su emplazamiento serían visibles prácticamente desde cualquier embarcación que recalase en la cala, ha sido explicado como un posible enclave relacionado con rituales de acción de gracias a la divinidad por su protección en los viajes marítimos (Orfila et al. 2010: 448-449).

La funcionalidad religiosa es también la que se atribuye al elemento central de las investigaciones desarrolladas por el citado Proyecto de Investigación, la Cova dels Jurats o de L'Esglesia, un santuario de tipo rupestre con dos espacios bien definidos. Hacia el exterior, en una de las varias repisas analizadas, aparece un amplio panel epigráfico ya publicado a principios del siglo XIX por J. Ramis (1817: 83-120) e incluido en el Corpus Inscriptionum Latinarum volumen II de 1886 ( $\mathrm{n}^{\circ}$. 37183724). Analizado de nuevo a mediados del siglo XX por C. Veny (Veny 1965: 160-170), ha sido recientemente objeto de una nueva lectura, que ha permitido reconocer un total de 29 inscripciones, que parecen estar asociadas a la celebración de los Parilia, y quizás también a los ludi Ceriales, fechándolas entre el 125 y el 230 d.C. aproximadamente (Orfila et al. 2013: 116).

Hacia el interior, el espacio está formado por una gruta de forma alargada, de aproximadamente $20 \mathrm{~m}$ de profundidad y $9 \mathrm{~m}$ de anchura, con diferentes plataformas laterales y estructura absidada (fig. 2). A la derecha del ábside se llevó a cabo en 2012 la excavación arqueológica de una cavidad en la roca en la que pudieron identificarse recortes, destinados posiblemente a recoger el agua que parece ser pudo manar en este punto, de lo que se deduce que tal vez este líquido elemento pudo tener alguna función en las actividades desarrolladas en la cueva.

En relación al cuerpo principal de la gruta, este se encuentra actualmente ocupado casi en su totalidad por el derrumbe de gran parte del techo, producido en un momento indeterminado de la historia, aunque claramente posterior a su uso en la Antigüedad. A pesar de ello, tras la caída de estos grandes fragmentos de piedra, quedaron libres dos espacios diferenciados aunque comunicados, básicamente un pasillo lateral y el fondo de la cueva. Ambos espacios han sido objeto de diferentes catas estratigráficas desarrolladas en los veranos de 2010, 2011 y 2012. Los trabajos de excavación llevados a cabo permitieron documentar la alternancia de UEs con un gran contenido en ceniza y elementos quemados, principalmente fauna, con otras que no presentaban este componente (Orfila et al. 2010: 457). Además parece que la mayor parte de los materiales aparecieron casi amontonados hacia el lateral este de la cueva, junto a la pared. Entre los materiales arqueológicos recuperados cabe destacar la presencia no solo de boles helenísticos a molde, objeto de este estudio, sino también un 
plato de pescado ebusitano, platos de cocina norteafricana, boles de barniz negro, numerosas lucernas (seguramente para la iluminación en el interior de la cueva) y cerámica talayótica a mano (Orfila et al. 2010: 457), contextualizados entre los siglos III-II a.C. y I-II d.C. (Orfila et al. 2010: 457) (fig. 3).

La presencia de numerosos cuencos y platos, mezclados con una importante cantidad de restos de fauna, ha llevado a proponer que en la cueva se llevase a cabo algún tipo de ritual religioso de tipo participativo, en el que debieron desempeñar un papel importante la comensalidad y el fuego (Orfila et al. 2010: 459).

\section{LAS CERÁMICAS MEGÁRICAS}

Tradicionalmente conocidas como "megáricas", estas cerámicas a molde con relieves - hemispherical moldmade relief bowls (Rotroff 2003: 91)-constituyen una de las producciones más características y populares del mundo helenístico. Producidas en diferentes puntos del Mediterráneo Oriental (Asia Menor y Grecia) y Central (Etruria/Lacio y Apulia) y reconocidas en multitud de yacimientos, presentan como forma más característica el cuenco, que en ocasiones sirvió como base para el cuerpo en la creación de boles con pie, cráteras e incluso formas cerradas tales como jarras o ánforas.

Estos cuencos suelen presentar una decoración en la que predominan eminentemente los temas vegetales, con un rico repertorio de hojas, rosetas, pámpanos o guirnaldas, aunque también son muy comunes los elementos de tipo geométrico e incluso figurado, conformando en ocasiones verdaderas escenas que han llegado a ser consideradas tributarias de la escultura en bulto redondo (Schmid 2006). En función de estos diferentes tipos de decoración, se suele hablar de boles tipo piña, imbricados, florales, figurados u homéricos.

Las investigaciones desarrolladas a lo largo del último siglo en torno a este tipo de producciones han permitido también la identificación de los distintos talleres que trabajaron de manera casi coetánea en los diferentes puntos del Mediterráneo, aunque por el momento la ciudad concreta en la que se ubicaron muchos de ellos resulta aún difícil de determinar. A pesar de la gran similitud en las formas y en los motivos decorativos, la mayoría de ellos profundamente estandarizados y generados a partir de moldes y punzones para los que se ha defendido una gran movilidad geográfica, son las pequeñas variantes y las diferencias en las combinaciones las que han permitido distinguir los diferentes talleres. Labor en la que resultó fundamental el monumental trabajo

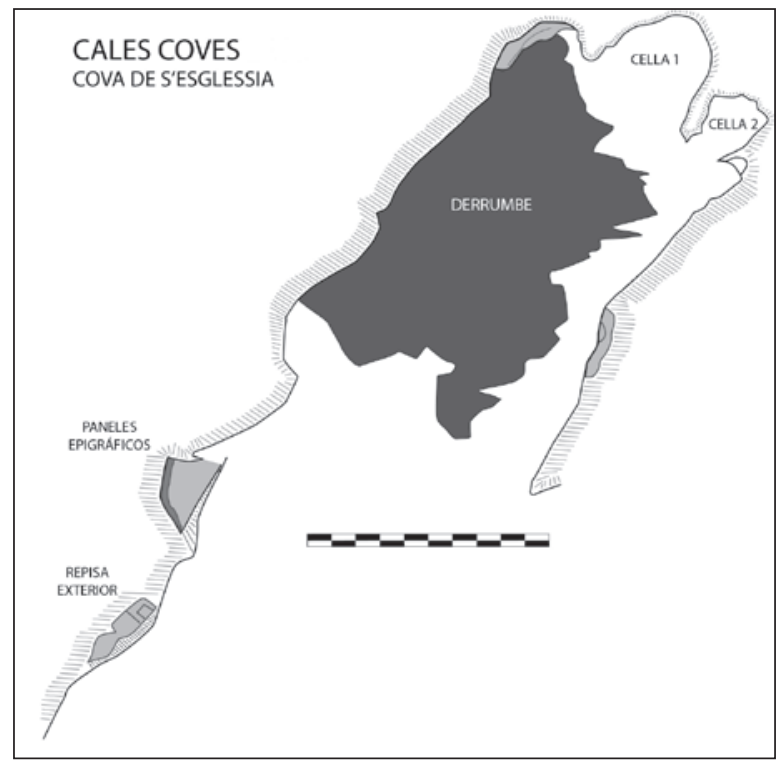

Figura 2. Planimetría de la Cova dels Jurats. Dibujo Mario Gutiérrez.

de Laumonier (1977), sistematizando las cerámicas de este tipo halladas en las excavaciones de Delos.

Con respecto a su cronología, parece ser que estas piezas comenzarían a fabricarse como imitación de vasos metálicos, tal vez más concretamente, como defiende Rotroff, imitando vasos de plata de procedencia alejandrina que habrían sido llevados a Atenas para la celebración de la primera Ptolemaia en esta ciudad en el 224 a.C. (Rotroff 2003: 92). En cualquier caso, sea o no esta la fecha exacta en la que estos boles con relieves comenzaron a fabricarse, los autores coinciden en fechar el inicio de estas producciones en la segunda mitad o el último cuarto del siglo III a.C. y su final a inicios del siglo I a.C. (Puppo 1995: 17; Schmid 2006: 46).

En relación a su hallazgo en Hispania, la mayor parte de los boles helenísticos con relieves localizados en la península proceden de talleres jonios, aunque también ha sido identificada la presencia de producciones itálicas e incluso locales (Vegas 1953-1954 y 1955-1956). De este modo, estas cerámicas, cuya comercialización se debe fechar aquí entre la segunda mitad del siglo II y mediados del siglo I a.C. (Cabrera 2004a: 8; Lara Vives 2004-2005: 123; Pérez Ballester 2012: 73), han sido identificadas en multitud de yacimientos, entre ellos Cartagena (Cabrera 1979; Lara Vives et al. 2009), Ampurias (Vegas 1953-1954 y $1955-$ 1956), la Alcudia de Elche (Cabrera 2004b; Lara Vives 2004-2005), El Monastil (Tordera Guarinos 1991), Pollentia (Arribas y Trías 1959), Ibiza (Fernández de 


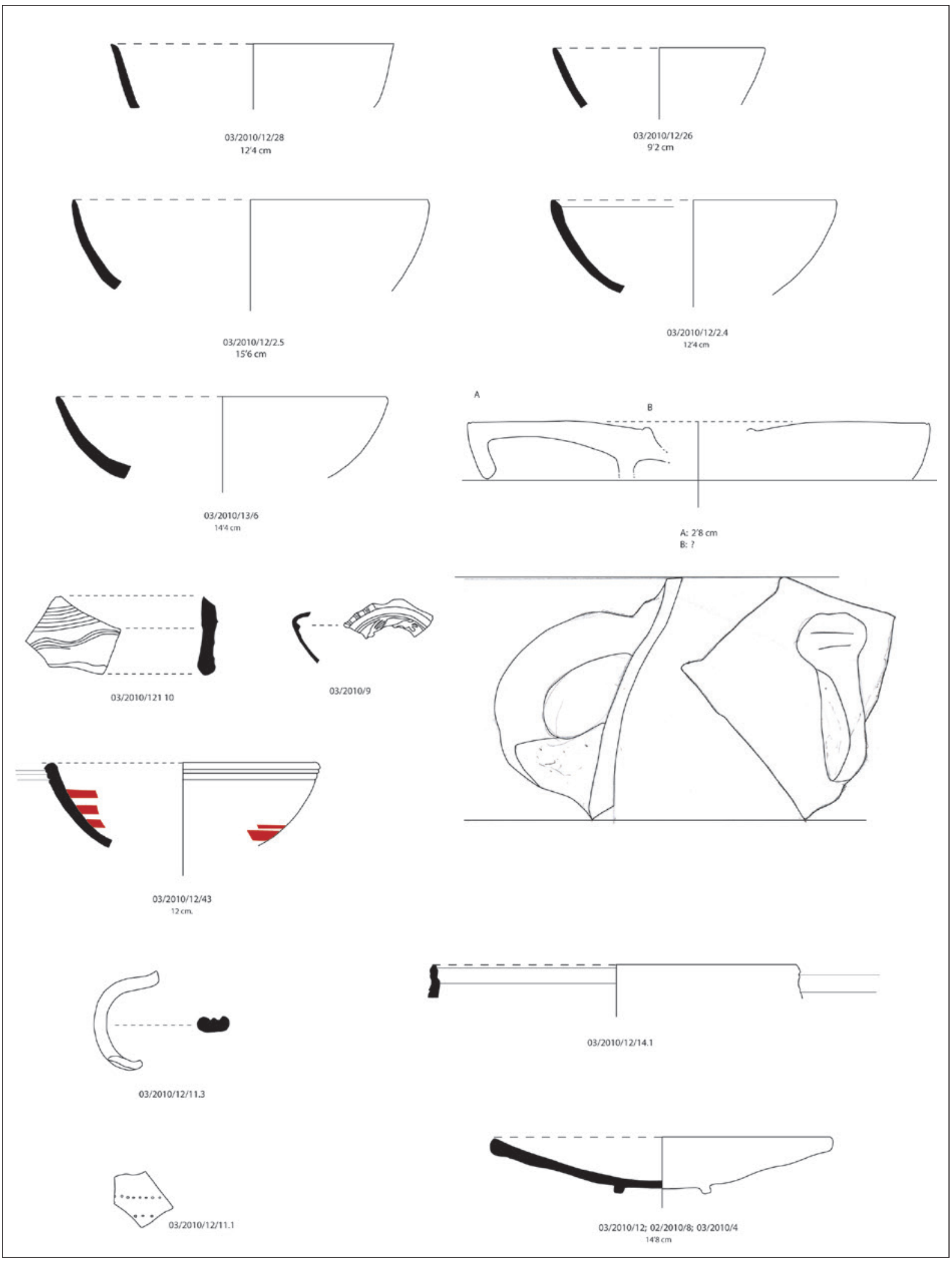

Figura 3. Selección de materiales cerámicos de la Cueva del Jurats. Dibujos de Purificación Marín. 
Avilés 1956 y 1957), o en varios yacimientos menorquines como los de Sa Torreta (Murray et al. 1934, lám. XXXIII, 32), Trepucó (Murray 1938: 21) y Torre Llafuda (Nicolás 1983, 233-234).

\section{CONTEXTO ARQUEOLÓGICO/ ESTRATIGRÁFICO DE LAS PIEZAS}

Los fragmentos de boles helenísticos con relieve hallados en las campañas desarrolladas en la Cova de l'Esglesia o dels Jurats pertenecen a las siguientes UEs (fig. 4):
- UEs 08 y 55: correspondientes al relleno de la escorrentía (en las catas 1, 2 y 3 ) en la que al parecer desecharon los materiales que cubrían los estratos de uso tras su limpieza. En esta zona de desecho se recuperó también cerámica púnica ebusitana, ánforas, lucernas, barnices negros (A), paredes finas, TSAA, cocina republicana y talayótica.

- UE 54: correspondiente a una fosa de furtivos documentada en la Cata 3. Entre los materiales recuperados destacan cerámica talayótica, cerámicas finas, sigillata clásica, lucernas y barnices $\operatorname{negros}(\mathrm{A})$.

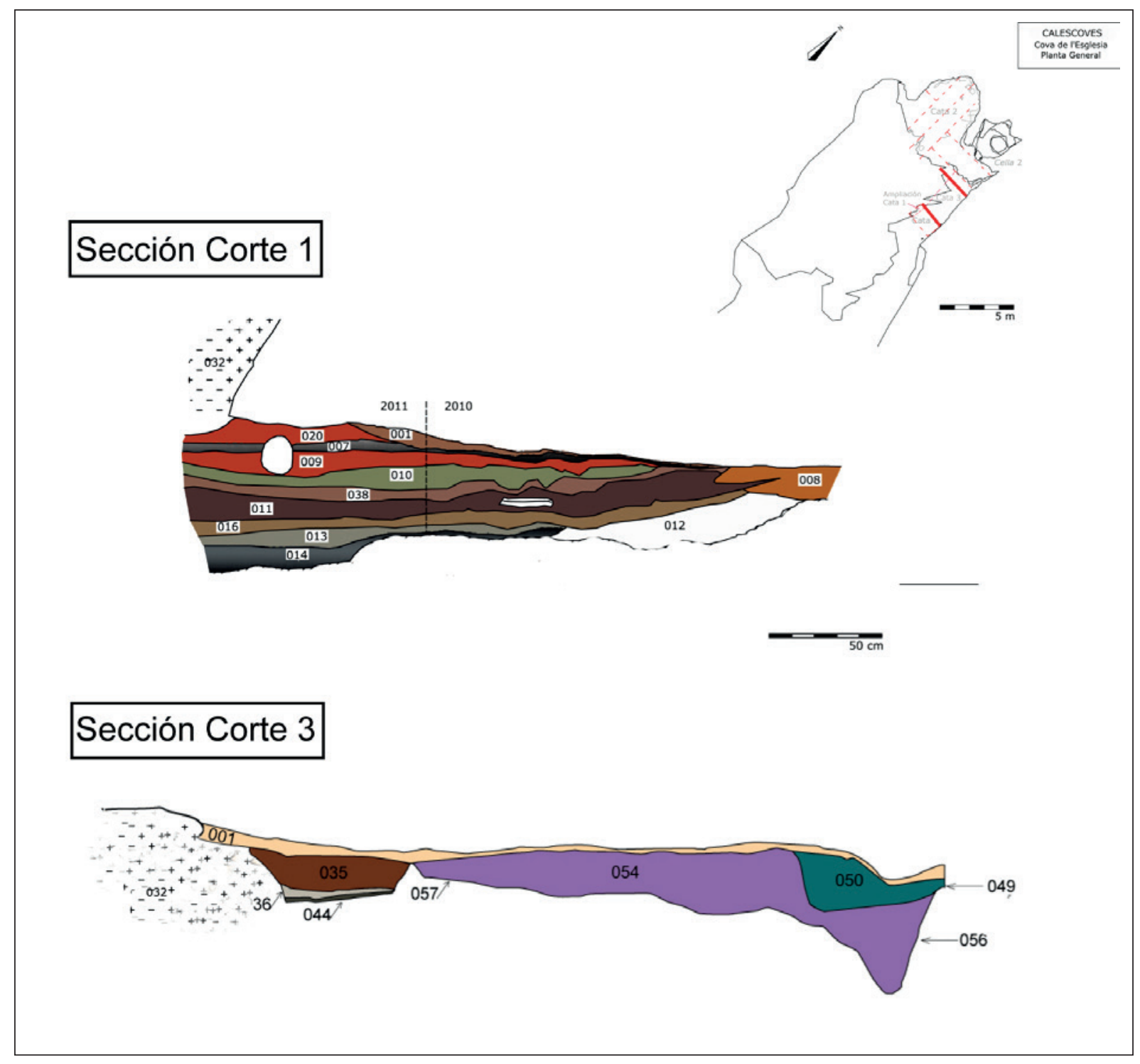

Figura 4. Secciones estratigráficas de la Cova del Jurats. Dibujos Elena Sánchez y Mario Gutiérrez. 
- UE 14 A: estrato en contacto directo con la roca en el que aparecieron también cerámicas talayóticas, lucernas y paredes finas

- UE 58: los fragmentos de boles helenísticos aparecen junto a cerámica talayótica, gris ampuritana, paredes finas, ánforas, cocina republicana, barnices negros (A) y TSAA.

\section{DECORACIONES Y PROCEDENCIA DE LAS CERÁMICAS HELENÍSTICAS CON RELIEVE DE CALESCOVES}

Como han indicado diversidad de autores, tal como señala Cabrera (2004), la mejor manera de establecer el taller y en consecuencia el lugar de origen de estas cerámicas helenísticas a molde es el estudio de sus decoraciones, que aunque muy estandarizadas, como ya hemos comentado, presentan determinadas diferencias en los punzones y su combinación que permiten llegar a algunas conclusiones al respecto (figs. 5 y 6).

En el caso de los fragmentos cerámicos recuperados en las excavaciones llevadas a cabo en Calescoves, los motivos decorativos pueden organizarse en cinco grupos bien diferenciados.

En primer lugar, lo que se ha dado en denominar "imbricados" -"folioles imbriquées" para Courby (1922) o "ecailles" para Laumonier (1977)-, un tipo de decoración formada por elementos vegetales, concretamente hojas, que se repiten ocupando la práctica totalidad del vaso y que podemos observar en los fragmentos 08/2012/01/7, 08/2012/54/7.4, 08/2012/54/7.5 y en uno

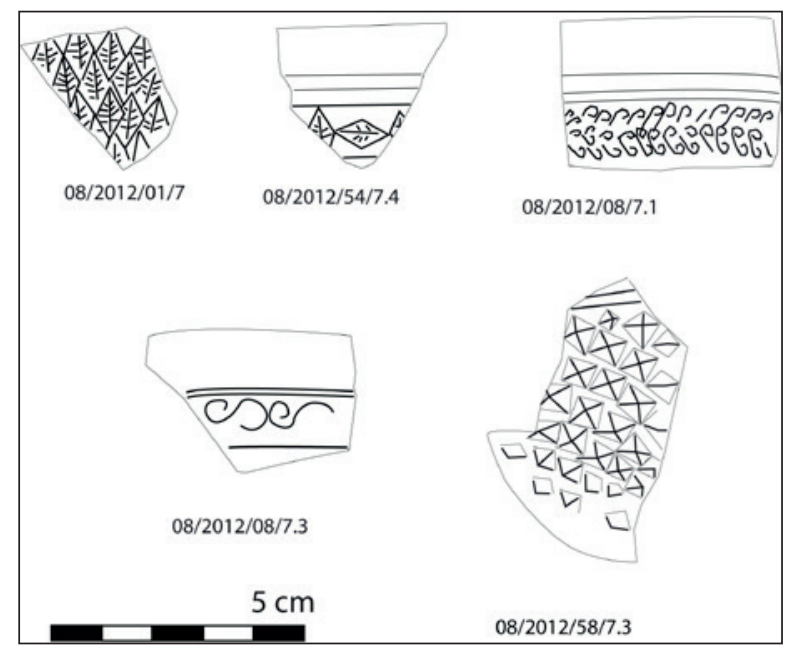

Figura 5. Decoraciones de los boles helenísticos a molde de la Cova dels Jurats. Dibujos Elena Sánchez. de los hallados por furtivos y publicados por Belén y Fernández Miranda (1979). En el caso concreto de los cuatro fragmentos que han sido recuperados en distintos momentos en el yacimiento, esta decoración se caracteriza por la forma lanceolada y extremadamente apuntada de las hojas, en las que la ramificación interior está formada por un nervio axial y nervios laterales oblicuos. Se trata de un motivo decorativo extremadamente recurrente en este tipo de vasos, pero que por sus características podemos situar entre las producciones procedentes de la zona de Jonia. Hojas imbricadas de tipología idéntica a las nuestras se pueden observar en talleres como los de Nénémakhos, Athènaios o Hèraios, entre otros (Laumonier 1977).

Bastante vinculado a este motivo decorativo se encuentra otro que hemos documentado ocupando la primera banda con decoración bajo el borde liso de cinco piezas-08/2012/54/7.1,08/2012/54/7.2,08/2012/54/7.3, 08/2012/55/7.1 y en uno de las halladas por furtivos y publicados por Belén y Fernández Miranda (1979)-. En este caso se trata de una banda simple formada por el mismo tipo de hojas que veíamos anteriormente -lanceoladas con nervio axial y nervios laterales oblicuos formando la ramificación interior-, pero que se combinan de manera que aparece una vertical seguida de otra en posición horizontal. No hemos podido localizar paralelos exactos para este tipo de banda decorativa, aunque un motivo muy similar pero con las hojas siempre en vertical o en horizontal aparece clasificado dentro de las series independientes de Leumonier, concretamente en el grupo VIb del conjunto de las "Six Séries" (Leumonier 1977: 396 y P1 93: 1203, 3071 y 2159).

El tercer motivo decorativo recurrente en los fragmentos de cerámica helenística a molde recuperados en las excavaciones en la Cova dels Jurats es la banda simple de $\mathrm{S}$ tumbadas dispuestas inmediatamente bajo el borde liso de los cuencos - fragmentos 08/2012/08/7.3, 08/2012/08/7.4 y 08/2012/54/7.6-. Estos “enroulements" (Courby 1992: fig. 76.12) son una clase de decoración muy común en las cerámicas de este tipo, por lo que resulta imposible determinar su procedencia.

Entre los grupos que hemos diferenciado, se encuentra también el formado por aquellos fragmentos que presentan una banda decorativa a base de chevrones con tres ojos - “chevrons triples oeillés" (Laumonier 1977)- bajo la banda lisa del borde. Varios de los fragmentos recuperados que presentaban este tipo de decoración, pertenecen a un vaso tipo piña que ha podido ser reconstruido (fig. 7) y que resulta idéntico al fragmento de cuenco que fue publicado por Belén y Fernández Miranda (1979: 34 fig. 13.2), aparecido 


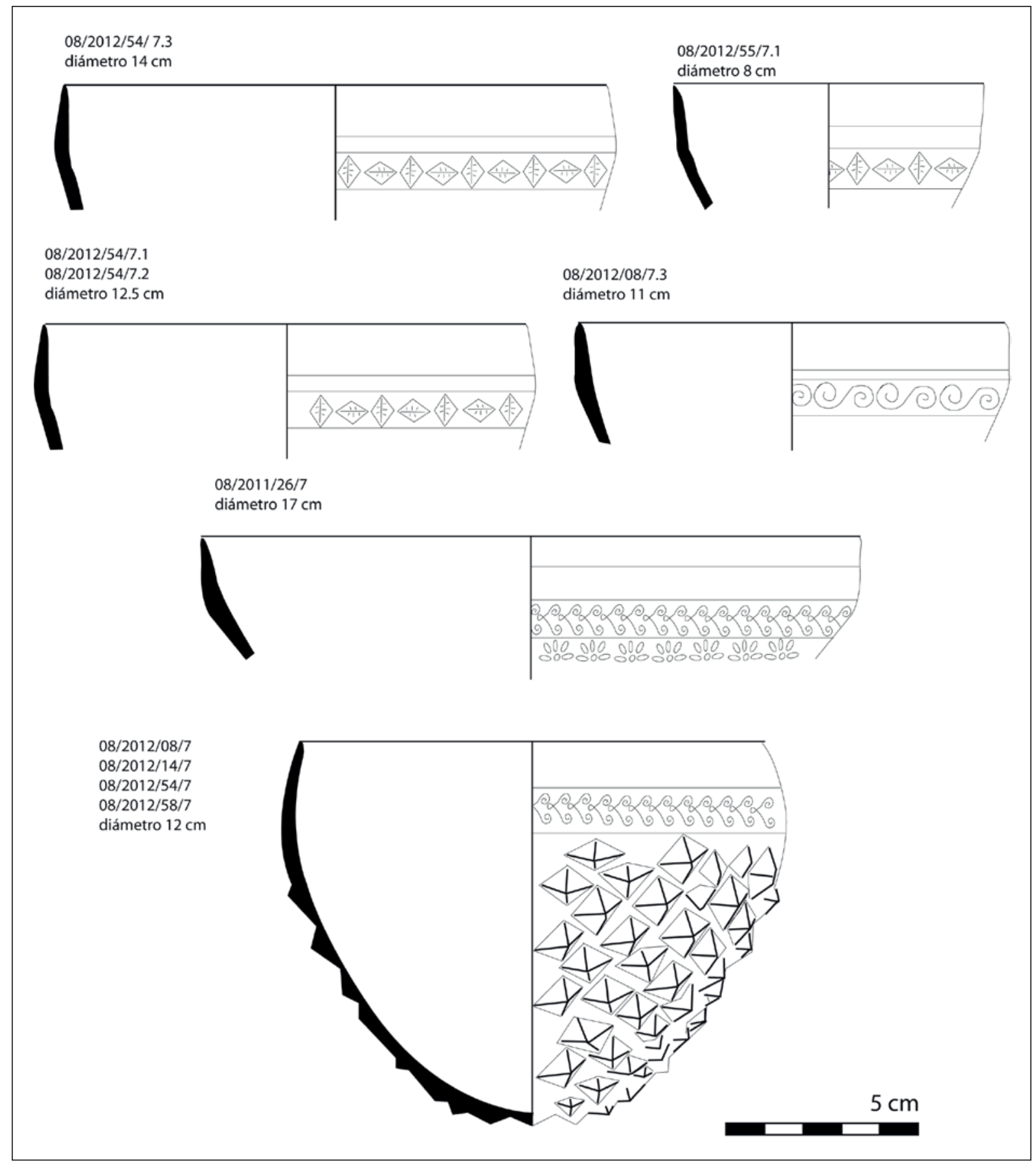

Figura 6. Boles helenísticos a molde de la Cova dels Jurats. Dibujos Elena Sánchez.

también en la cueva como resultado de la intervención de furtivos. Este hecho nos lleva a plantar la posibilidad de que, si la excavación de los furtivos en la que se hallaron dichos fragmentos es aquella de la que se han podido reconstruir los límites durante nuestros trabajos en la Cata 3, podríamos estar ante fragmentos del mismo cuenco tipo piña y no ante dos cuencos diferentes.

Este tipo de cuencos tipo piña o "bols à bossettes" (Courby 1922: fig. 80.6), del que tenemos varios fragmentos en la Cova dels Jurats -08/2012/08/7 (1, 2 y 5), 08/2012/14A.7, 08/2012/54/7.7, 08/2012/58/7 (1, 2 y 3 ), 


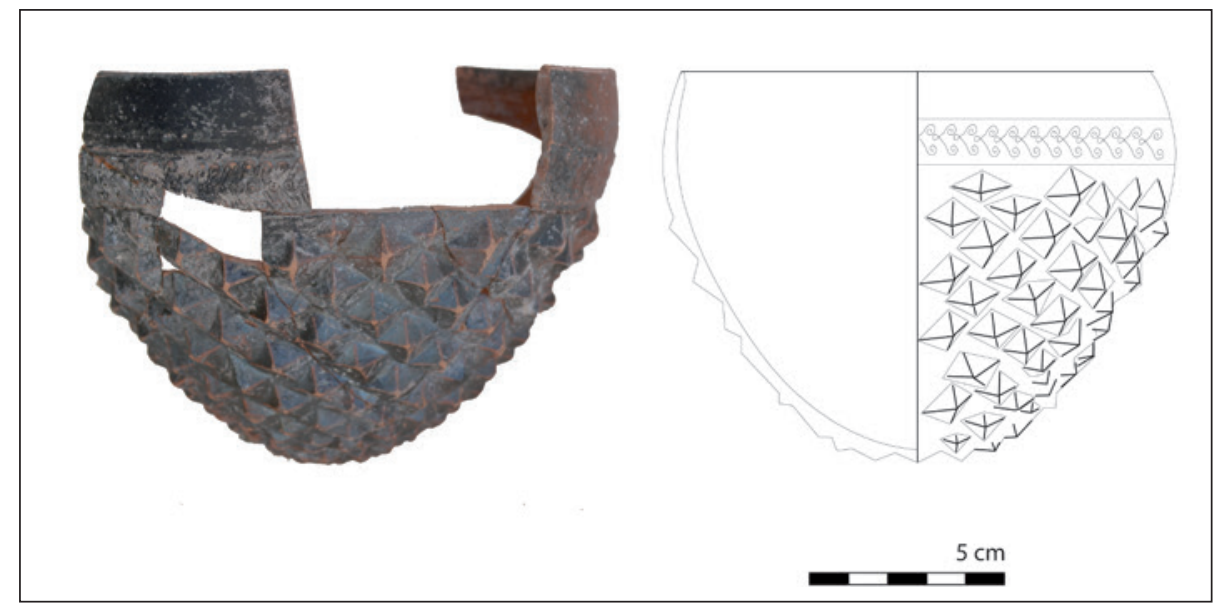

Figura 7. Cuenco tipo piña de la Cova dels Jurats. Elena Sánchez. y el ya publicado anteriormente (Belén y Fernández Miranda 1979) - es en palabras de Rostroff el más sencillo de todos, siendo el molde realizado en ocasiones directamente desde una auténtica piña, por lo que como resultado pueden presentar una forma diferente a la del resto de los cuencos, es decir, más estrechos y alargados y con la base redondeada (Rostroff 1982: 16). Este tipo de boles, aunque no parecen ser demasiado comunes, de hecho existen pocos ejemplos en la Península Ibérica $^{1}$, parece que fueron fabricados en diferentes talleres, tanto en aquellos definidos como jonios (Laumonier 1977), como en la misma Atenas (Rotroff 1982), lo que hace difícil determinar el origen exacto de nuestra pieza, pues no hemos encontrado ningún paralelo exacto para la misma.

Sin embargo, el motivo de los chevrones con tres ojos que aparece en la banda decorativa justo por debajo del borde liso de la pieza 08/2012/58/7.1 -y que también aparece en los fragmentos 08/2012/08/7.1, 08/2012/08/7.2, 08/2012/54/7.7, y 08/2012/58/7.2constituye una de las decoraciones atribuidas al conocido como Taller del Monograma (Laumonier 1977: Pl $45 n^{\circ} 1649$ y Pl $46 n^{\circ}$ 1777). Al no haber encontrado este tipo de decoración entre las piezas atribuidas a otros talleres, podemos concluir que tanto el cuenco tipo piña como el resto de los fragmentos de borde con chevrones de tres ojos (pertenezcan o no a otro cuenco similar) procederían de piezas fabricadas en este taller, que las últimas investigaciones sitúan concretamente en Éfeso (Schmid 2006: 48).

A estos grupos habría que añadir un fragmento totalmente diferente al resto, publicado por Belén $\mathrm{y}$

1. Sólo hemos podido localizar referencias a la existencia de tres boles tipo piña, citados por Pérez Ballester (1994: 353).
Fernández Miranda (1979) y procedente de las excavaciones subacuáticas en el fondeadero de Calescoves. Esta pieza presenta por un lado una banda de "perles et pirouettes" (Courby 1922: fig. 76.5) o astrágalos, un motivo muy común en estas producciones, para el que no hemos podido localizar paralelos exactos pues no hemos hallado ninguna otra pieza en la que los astrágalos presentaran una forma tan angular, pues son mucho más comunes aquellos que presentan un dibujo más curvilíneo o en el caso de ser romboidales, estos son bastante más alargados. Por otro lado, la pieza cuenta también con una banda de ovas y dardos, de nuevo un motivo muy recurrente y estandarizado en estas cerámicas, por lo que ha sido imposible, en base al dibujo publicado, determinar el taller de origen.

El estudio pormenorizado de los boles helenísticos con relieve a molde hallados en las excavaciones desarrolladas en la Cova dels Jurats de Calescoves, permite concluir, aunque no hayamos encontrado paralelos exactos para las piezas aquí recuperadas, en gran medida debido al reducido tamaño de la mayor parte de los fragmentos, que los vasos fueron producidos seguramente en talleres ubicados en la zona jonia. Habiendo identificado con seguridad solamente uno de estos talleres, el taller del Monograma, que por otra parte resultó ser el más prolífico de entre los que trabajaron en aquella región y del que salieron la mayor parte de las piezas que pueden hallarse en el Mediterráneo (Cabrera 2004: 65; Schmid 2006: 49). Parece que este es también el caso en la península ibérica, pues han sido identificadas producciones atribuidas a este taller en Elche (Cabrera 2004; Lara Vives 2004-2005), Cartagena (Cabrera 1979; Lara Vives et al. 2009), Valentia (Montesinos 1983) y El Monastil (Tordera Guarinos 1991). 
Con respecto a las fechas exactas en las que estuvo en funcionamiento el Taller del Monograma, los estudios tradicionales han defendido una cronología que va desde mediados del siglo II a mediados del siglo I a.C. (Laumonier 1977; Cabrera 2004), aunque con un floruit que habría que situar en los últimos años del siglo II a.C. (Cabrera 1979: 103-104; Cabrera 2004: 65), o de manera más amplia en la segunda mitad del siglo (Schmid 2006: 49). Según Schmid, los boles tipo piña y aquellos con decoraciones de hojas imbricadas se encontrarían entre las producciones más antiguas de este taller, y por lo tanto entre aquellas que habría que fechar en el segundo o tercer cuarto de ese siglo II a.C. (Schmid 2006: 49).

\section{CONCLUSIONES}

Una cuestión interesante en relación al origen de estas cerámicas helenísticas a molde, es tratar de dilucidar la vía que siguieron hasta llegar a la península ibérica y las Baleares. Los últimos estudios coinciden en descartar la existencia de un contacto directo entre ambos extremos del Mediterráneo para el comercio de este tipo de producciones, basando la llegada de estas hasta este sector más occidental del Mediterráneo a través de intermediarios (Bats 1979: 164; Pérez Ballester 1994; Jaeggi 2000: 199). Siendo la teoría más aceptada la defendida entre otros por Pérez Ballester, que asocia la llegada de diferentes cerámicas como estos boles de relieve o los lagynoi de engobe blanco, entre la segunda mitad del siglo II a.C. y el primer tercio del siglo I a.C., con la redistribución de vino rodio, y las correspondientes ánforas, por parte de comerciantes itálicos del área de Nápoles-Pozzuoli. Materiales que llegarían a estas costas procedentes de Delos, donde desde el 166 a.C. se habían asentado numerosos comerciantes itálicos, y que serían incluidos en el comercio de vinos itálicos y cerámicas campanienses con Iberia/Hispania (Pérez Ballester 1994 y 2012).

Sin embargo, una cuestión complementaria a esta sería determinar si los intermediarios responsables de la distribución de estos materiales por las Baleares fueron también estos comerciantes itálicos, o si por el contrario en este área la redistribución de estos materiales debe ser atribuida más bien a los comerciantes ebusitanos, como podría derivarse de la importante presencia de material púnico-ebusitano presente tanto en el registro material de las últimas excavaciones de la Cova dels Jurats, como en el catálogo publicado de las excavaciones llevadas a cabo en el fondeadero de Calescoves (Belén y Fernández-Miranda 1979). Una posibilidad, la de la redistribución por parte de comerciantes ibicencos de materiales procedentes del Mediterráneo Oriental, que ya había sido apuntada para explicar la presencia de numerosos grafitos púnicos en los materiales procedentes del pecio de El Sec (Arribas et al. 1987: 655), fechado sin embargo dos siglos antes de la cronología propuesta para los boles helenísticos aquí analizados.

Un último elemento que resta por analizar es el de la función que este tipo de vasos desempeñó en la Cova del Jurats. Su relación con el comercio de vino griego, como se desprende de su ya citada frecuente relación con ánforas rodias y lagynoi de engobe blanco, ha llevado a Pérez Ballester a proponer que estos boles tendrían en el extremo occidental del Mediterráneo la misma función con la que habían sido concebidos en sus lugares de origen, el consumo de vino, probablemente de vino griego (Pérez Ballester 2012: 74). Sin embargo, la frecuente aparición de estos vasos cerámicos en contextos cultuales, cuestión sobre la que ha llamado la atención Semeraro en relación al registro de Herapolis y Iasos (Semeraro 2005), y funerarios (Pérez Ballester 2012: 75), permite plantear la posibilidad de dar un paso más en la interpretación de su uso en contextos como el excavado en Calescoves, y asociar directamente estos cuencos con los rituales y libaciones que se pudieron llevar a cabo en la cueva. Contexto de uso al que deben vincularse sin duda también otros vasos de pequeño tamaño recuperados en las excavaciones de la Cova dels Jurats, tanto cerámicas de tradición talayótica como importaciones (barnices negros y paredes finas entre otros). 


\section{APÉNDICE \\ CATÁLOGO DE FRAGMENTOS DE BOLES HELENÍSTICOS CON RELIEVE A MOLDE HALLADOS EN EL CONJUNTO ARQUEOLÓGICO DE CALESCOVES}

\section{Materiales procedentes de las últimas excavaciones en Calescoves}

Referencia: 08/2012/01/7

Descripción: Fragmento de cuenco decorado con hojas imbricadas de forma lanceolada con nervio axial y nervios laterales oblicuos formando la ramificación interior.

Arcilla y barniz de color marrón.

Localización: Cova dels Jurats. Cata 3. UE 01

\section{Referencia: 08/2012/08/7.3}

Descripción: Borde de cuenco. El fragmento conserva una primera banda lisa, bajo la cual aparece otra decorada con $\mathrm{S}$ tumbadas.

Pasta de color anaranjado y barniz gris oscuro o negro.

Localización: Cova dels Jurats. Cata 3. UE 08

\section{Referencia: 08/2012/08/7.4}

Descripción: Fragmento de cuenco. Conserva una primera banda lisa, bajo la cual aparece otra decorada con $\mathrm{S}$ tumbadas.

Pasta de color anaranjado y barniz gris oscuro o negro.

Localización: Cova dels Jurats. Cata 3. UE 08

Referencia: 08/2012/08/7.1

Descripción: Borde de cuenco. El fragmento conserva una primera banda lisa, bajo la cual aparece otra decorada con chevrones con tres ojos.

Pasta y barniz de color anaranjado.

Localización: Cova dels Jurats. Cata 3. UE 08

Referencia: 08/2012/08/7.2

Descripción: Borde de cuenco. El fragmento conserva una primera banda lisa, bajo la cual aparece otra decorada con chevrones con tres ojos.

Pasta anaranjada y barniz de color gris oscuro o negro.

Localización: Cova dels Jurats. Cata 3. UE 08

Referencia: 08/2012/08/7.5

Descripción: Fragmento del cuerpo de un cuenco tipo piña caracterizado por la decoración a base de prominencias de forma piramidal.

Pasta anaranjada y barniz grisáceo.

Localización: Cova dels Jurats. Cata 3. UE 08
Referencia: 08/2012/14A/7

Descripción: Fragmento del cuerpo de un cuenco tipo piña caracterizado por la decoración a base de prominencias de forma piramidal.

Pasta anaranjada y barniz grisáceo.

Localización: Cova dels Jurats. Cata 3. UE 14A

Referencia: 08/2012/54/7.6

Descripción: Fragmento. Solamente se conserva parte de una banda decorativa formada por $\mathrm{S}$ tumbadas.

Pasta de color marrón y barniz negro.

Localización: Cova dels Jurats. Cata 3. UE 54

Referencia: 08/2012/54/7.4

Descripción: Fragmento de cuenco decorado con hojas imbricadas, de forma lanceolada con nervio axial y nervios laterales oblicuos formando la ramificación interior.

Arcilla de color anaranjado y barniz negruzco.

Localización: Cova dels Jurats. Cata 3. UE 54

Referencia: 08/2012/54/7.5

Descripción: Fragmento de cuenco decorado con hojas imbricadas, de forma lanceolada con nervio axial y nervios laterales oblicuos formando la ramificación interior.

Arcilla de color anaranjado y barniz negruzco.

Localización: Cova dels Jurats. Cata 3. UE 54

Referencia: 08/2012/54/7.3

Descripción: Borde de cuenco. El fragmento conservado presenta una primera banda lisa, la del borde propiamente dicho, y debajo una decorada con hojas lanceoladas. Las hojas aparecen en una fila simple combinando una colocada en posición horizontal y otra en vertical.

Arcilla anaranjada y barniz de color gris oscuro o negro. Localización: Cova dels Jurats. Cata 3. UE 54

Referencia: 08/2012/54/7.1

Descripción: Borde de cuenco. El fragmento conservado presenta una primera banda lisa, la del borde propiamente dicho, y debajo una decorada con hojas de lanceoladas con nervio axial y nervios laterales oblicuos formando la ramificación interior. Las 
hojas aparecen en una fila simple combinando una colocada en posición horizontal y otra en vertical. Arcilla y barniz de color marrón.

Localización: Cova dels Jurats. Cata 3. UE 54

\section{Referencia: 08/2012/54/7.2}

Descripción: Borde de cuenco. El fragmento presenta una primera banda lisa, la del borde propiamente dicho, y debajo una decorada con hojas de lanceoladas que aparecen en una fila simple combinando una colocada en posición horizontal y otra en vertical.

Arcilla y barniz de color marrón.

Localización: Cova dels Jurats. Cata 3. UE 54

\section{Referencia: 08/2012/54/7.7}

Descripción: Fragmento. Restos de una banda decorada con chevrones con tres ojos.

Pasta anaranjada y barniz de color gris oscuro.

Localización: Cova dels Jurats. Cata 3. UE 54

\section{Referencia: 08/2012/55/7.1}

Descripción: Borde de cuenco. El fragmento conservado presenta una primera banda lisa, la del borde propiamente dicho, y debajo una decorada con hojas lanceoladas con nervio axial y nervios laterales oblicuos formando la ramificación interior. Las hojas aparecen en una fila simple combinando una colocada en posición horizontal y otra en vertical.

Arcilla anaranjada y barniz de color negro brillante.

Localización: Cova dels Jurats. Cata 3. UE 55

Referencia: 08/2012/58/7.2

Descripción: Borde de cuenco. El fragmento conserva una primera banda lisa, bajo la cual aparece otra decorada con chevrones con tres ojos.
Pasta y barniz de color anaranjado.

Localización: Cova dels Jurats. Cata 3. UE 58

Referencia: 08/2012/58/7.1

Descripción: Cuenco tipo piña (varios fragmentos). La decoración se organiza en una primera banda lisa, bajo la cual aparece otra con chevrones con tres ojos. El resto del cuerpo del cuenco está decorado con prominencias de forma piramidal. El cuenco presenta una base redondeada.

Pasta anaranjada y barniz grisáceo.

Localización: Cova dels Jurats. Cata 3. UE 58

Referencia: 08/2012/58/7.3

Descripción: Fragmento de cuenco tipo piña. Se aprecian la existencia de dos espacios decorativos: uno superior del que quedan pocos restos, por lo que no se puede especificar la decoración, y otro que ocupa el resto del cuerpo del cuenco y que está decorado con prominencias de forma piramidal. El cuenco presenta una base redondeada.

Pasta anaranjada y barniz grisáceo.

Localización: Cova dels Jurats. Cata 3. UE 58

\section{Materiales procedentes de Calescoves publicados con anterioridad (fig. 8)}

Descripción: Borde de cuenco que presenta una primera banda lisa, la del borde propiamente dicho, y debajo una decorada con hojas lanceoladas con nervio axial y nervios laterales oblicuos formando la ramificación interior. Las hojas aparecen en una fila simple combinando una colocada en posición horizontal y otra en vertical.

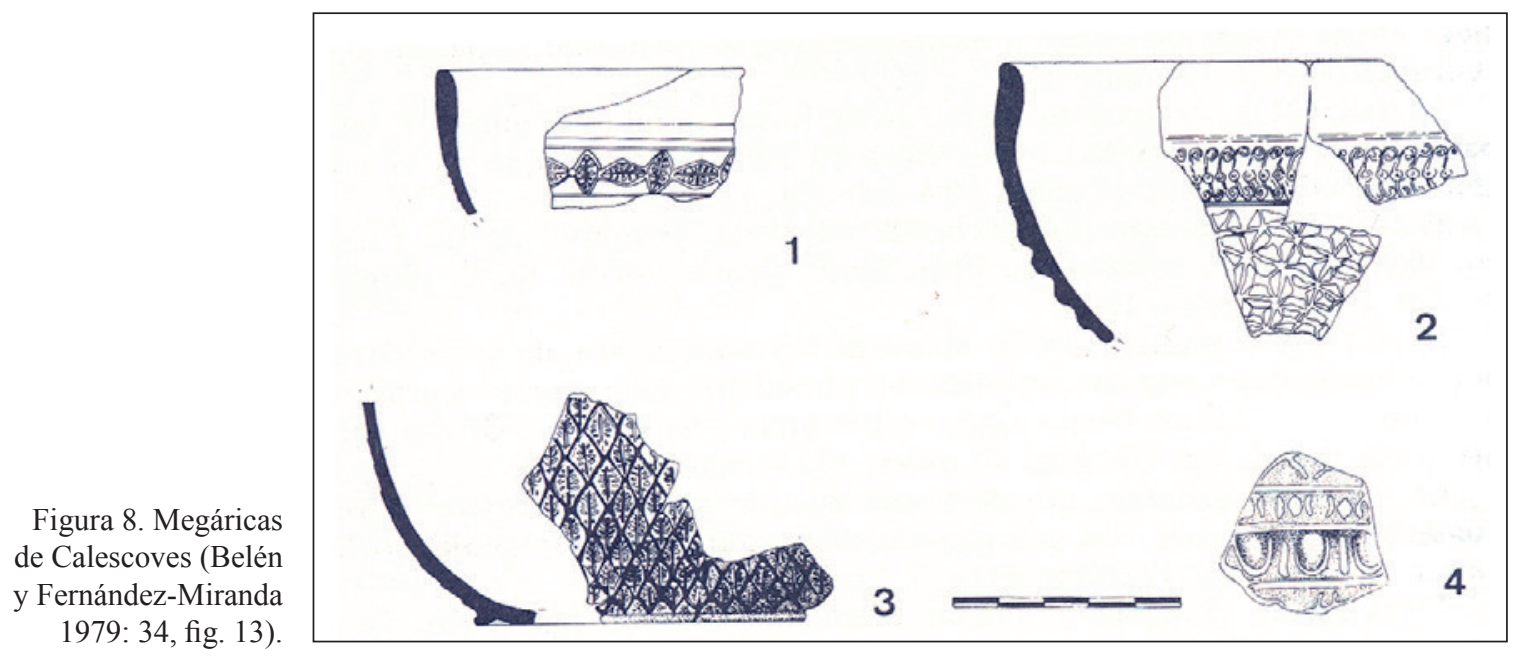


Localización: Cova dels Jurats. Furtivos

Bibliografia: Belén y Fernández-Miranda 1979: 34, fig. 13.

Descripción: Cuenco tipo piña. La decoración se organiza en una primera banda lisa, bajo la cual aparece otra con chevrones con tres ojos. El resto del cuerpo del cuenco está decorado con lo que parece ser algún tipo de prominencias. El cuenco presenta una base redondeada.

Localización: Cova dels Jurats. Furtivos.

Bibliografia: Belén y Fernández-Miranda 1979: 34, fig. 13.

Descripción: Fragmento de paredes y base de cuenco decorado con hojas imbricadas, de forma lanceolada con nervio axial y nervios laterales oblicuos formando la ramificación interior.

Procedencia: Cova dels Jurats. Furtivos.

Bibliografia: Belén y Fernández-Miranda 1979: 34, fig. 13.

Descripción: Fragmento de cuenco. Se observa la existencia de al menos cuatro bandas decorativas, siendo difícil de precisar los motivos que ocupaban la primera y la cuarta. Por su parte, la segunda está formada por una hilera de astrágalos, presentando el cuerpo de forma romboidal. La tercera está compuesta por ovas y dardos.

Localización: Fondeadero de Calescoves.

Bibliografia: Belén y Fernández-Miranda 1979: 34, fig. 13.

\section{Agradecimientos}

Este trabajo es una contribución al proyecto "Intervención arqueológica en Calescoves (Alaior, Menorca). Estudio epigráfico y revisión del lugar como santuario: Cova dels Jurats o de l'Esglesia y Cobertxo Blanc", dirigido por Margarita Orfila, Marc Mayer y Giulia Baratta.

\section{BIBLIOGRAFÍA}

Arribas, A. y Trías De Arribas, G. (1959): “Cerámica de "Megara" en Pollentia (Alcudia, Mallorca)". Excavaciones Arqueológicas en España XXXII: 8492. Madrid.

Arribas, A.; Trías, M.G.; Cerda, D. y De la Hoz, J. (1987): El barco de El Sec (Calvià, Mallorca). Estudio de los materiales. Mallorca.

Bats, M. (1979): "Bols hellénistiques à reliefs trouvés à Olbia en Ligurie (Hyères, Var)". Revue archéologique de Narbonnaise 12: 161-172.
Belén, M. y Fernández-Miranda, M. (1979): El fondeadero de Cales Coves (Menorca, Islas Baleares). Excavaciones Arqueológicas en España 101. Madrid.

Cabrera Bonet, P. (1979): "La cerámica helenística de relieves de Cartagena". Cuadernos de Prehistoria y Arqueología 5-6: 81-104.

Cabrera, P. (2004a): "Vasos cerámicos de importación de lujo del Mediterráneo oriental y central”, en R. Olmos Romera y P. Rouillard (eds.), La vajilla ibérica en época helenística: (siglos IV-III al cambio de era), Seminario celebrado en la Casa de Velázquez (2223 de enero de 2001): 5-18. Madrid (2001), Madrid.

Cabrera, P. (2004b): "La cerámica helenística de relieves de la Alcudia", en T. Tortosa (ed.), El yacimiento de La Alcudia (Elche, Alicante): pasado y presente de un enclave ibérico, Anejos de Archivo Español de Arqueología: 55-69. Madrid.

Courby, F. (1922): Les vases grecs a reliefs. París.

Fernández de Avilés, A. (1956): “Cuenco megárico de Ibiza en el Museo Arqueológico de Madrid”, en Actas del Ir Congreso de Estudios Clásicos: 296-300. Madrid.

Fernández de Avilés, A. (1957): “Cerámica de Megara en Espanha”. Revista Guimarães LXVII: 47-54.

Fernández-Miranda, M. (1977): “Arqueología submarina de Menorca", en XIV Congreso Nacional de Arqueología: 811-826. Zaragoza.

Fernández-Miranda, M. y Rodero, A. (1991): “Arqueología subacuática en Baleares", en Jornadas de arqueología subacuática en Asturias: 133-145. Oviedo.

Gornes, S. (1997): “Arqueología de la muerte y cambio social. Análisis e interpretación de la necrópolis de Cales Coves, Menorca”. Complutum 7: 91-103.

Gornes, S. (2000): "Ipogei del Talaiotico finale: analisi e interpretazione della necrópolis di Cales Coves, Minorca", en L'ipogeismo nel Mediterráneo: svilippo, quadri culturali vol. II: 553-571.

Gornés, S. y Gual, J. (2000): "El hipogeo XXI de la necrópolis de Cales Coves, Minorca", en L'ipogeismo nel Mediterráneo:origini, sviuippo, quadri culturali vol. II: 573-590. Sassari.

Jaeggi, O. (2000): Der Hellenismus auf der Iberischen Halbinsel: Studien zur iberischen Kunst und Kultur : das Beispiel eines Rezeptionsvorgangs. Mainz am Rhein.

Lamounier, A. (1977): La céramique hellénistique à reliefs. I Les ateliers “ioniens". École Francaise d'Athenes et Rome, París.

Lara Vives, G. (2004-2005): “Cerámicas helenísticas de relieves en La Alcudia (Elche, Alicante)". Lucentum XXIII-XXIV: 105-126. 
Lara Vives, G.; Mendiola Tébar, E. M. y López Seguí, E. J. (2009): “Un cuenco de cerámica helenística de relieves procedente de la villa romana Huerta del Paturro (Cartagena)". Mastia 8: 35-41.

Montesinos Martinez, J. (1983): “Constatación de cerámica helenística de relieves en Valencia”. Arse 18: 367-371.

Murray, M. A. (1934): Cambridge Excavations in Minorca. Sa Torreta. Londres.

Murray, M.A. (1938): Cambridge Excavations in Minorca. Trapucó, part II. Londres.

Nicolas, J. de (1983): "Romanización de Menorca", en Geografia e Historia de Menorca, tomo IV: 201283. Ciudadela.

Orfila, M.; Baratta, G. y Mayer, M. (2010): "Los santuarios de Calescoves (Alaior, Menorca): Coberxo blanc y Cova dels Jurats o de L'Esglesia. Informe Preliminar". Cuadernos de prehistoria y Arqueología de la Universidad de Granada 20: 439-477.

Orfila, M.; Baratta, G. y Mayer, M. (2013): "El santuario de Calescoves (Alaior, Menorca): la Cova dels Jurats o Església", V Jornades d'arqueologia de les Illes Balears: 109-117. Palma.

Pérez Ballester, J. (1994): “Asociaciones de laginos, boles helenísticos de relieves y ánforas rodias en contextos mediterráneos (siglos II y I aC)", en Iberos y griegos: lecturas desde la diversidad, Huelva Arqueológica XIII.2: 345-365.

Pérez Ballester, J. (2012): “Sobre cerámicas Helenísticas en Iberia/Hispania. Significado y funcionalidad". Archivo Español de Arqueología 85: 65-78.

Plantalamor, L. (1991): "Los asentamientos costeros de la isla de Menorca", en Atti del II Congreso Internazionale di Studi Fenici i Punici III: 1151-1160. Roma.

Puppo, P. (1995): Le coppe megaresi in Italia. Roma.
Ramis Ramis, J. (1817): Inscripciones romanas que existen en Menorca, y otras relativas à la misma sacadas de varios escritores; suplidas é ilustradas en quanto se ha podido (Edición facsímil, ed. Nura, Menorca 1995).

Rotroff, S. I. (1982): Hellenistic Pottery. Athenian and Imported Moldmade Bowls. Princeton, N.J.

Rotroff, S. I. y Oliver, A. (2003): The hellenistic pottery from Sardis: the finds through 1994. Archaeological Exploration of Sardis, Monograph 12. Cambridge, Mass.

Sánchez López, E.; Gutiérrez Rodríguez, M. y Orfila Pons, M. (2013): “Los asentamientos costeros de Menorca: el caso de Es Castellet (Calescoves, Alaior)", V Jornades d'Arqueologia de les Iles Balears: 59-67. Palma.

Schmid, S. G. (2006): Boire pour Apollon. Céramique hellénistique et banquets dans le Sanctuaire d'Apollon Daphnéphoros. ERETRIA XVI. Fouilles et recherches. Lausana, École Suisse d'Archéologie en Grèce.

Semeraro, G. (2005): "Per un approccio contestuale alla lettura delle immagni. Le ceramiche a rilievo di Hierapolis di Frigia". Mélanges de l'École Française de Rome. Antiquité 117.1: 83-98.

Vegas, M. (1953-1954): "Dos vasos megáricos de Ampurias". Ampurias XV-XVI: 352-355

Vegas, M. (1955-1956): "Fragmento de molde megárico de Ampurias". Ampurias XVII-XVIII: 252

Veny Melià, C. (1965): Corpus de las inscripciones baleáricas hasta la dominación árabe. Biblioteca de la Escuela Española de Historia y Arqueología en Roma, 15. Roma.

Veny Melià, C. (1982): La necrópolis protohistórica de Cales Coves, Menoría. Biblioteca Praehistorica Hispana 20. Madrid. 